\title{
Einen Forschungsaufenthalt in Frankreich vorbereiten
}

Niels F. May

\section{OpenEdition}

\section{Journals}

Édition électronique

URL : http://journals.openedition.org/ifha/7914

DOI : $10.4000 /$ ifha. 7914

ISSN : 2198-8943

\section{Éditeur}

IFRA - Institut franco-allemand (sciences historiques et sociales)

Référence électronique

Niels F. May, "Einen Forschungsaufenthalt in Frankreich vorbereiten », Revue de l'IFHA [Online], HS | 2014, Online erschienen am: 01 September 2014, aufgerufen am 01 Mai 2019. URL : http:// journals.openedition.org/ifha/7914; DOI : 10.4000/ifha.7914

Ce document a été généré automatiquement le 1 mai 2019.

(CIFHA 


\title{
Einen Forschungsaufenthalt in Frankreich vorbereiten
}

\author{
Niels F. May
}

\section{Einleitung}

1 Wesentlich für die Planung eines Forschungsaufenthalts ist eine möglichst genaue Bestimmung seines Zwecks: Handelt es sich um eine kurze Archivreise zur Ergänzung der bereits erarbeiteten Forschungsergebnisse? Um eine ausgiebige Archivrecherche? Oder um einen längerfristigen Aufenthalt, der nicht nur dem Quellenstudium dient, sondern auch mit der französischen Universitäts- und Forschungslandschaft vertraut machen soll? Außerdem sollte man sich fragen, auf welche Formen institutioneller Unterstützung zurückgegriffen werden kann (zum Beispiel im Rahmen der von der DFH getragenen strukturierten binationalen Master- und Promotionsstudiengänge) ${ }^{1}$ ?

In Frankreich können in Archiven und Bibliotheken - wie häufig auch hierzulande längere Wartefristen den Zugang zu bestimmten Archivbeständen erschweren. Die jährlichen Schließungsperioden oder gerade anstehende Renovierungen und Umbauten können die Arbeit behindern. Daher empfiehlt es sich, die Webseiten der jeweiligen Institutionen im Voraus zu besuchen. Auch der deutlich unterschiedliche Semester- bzw. Jahresrhythmus des französischen Universitätslebens muss berücksichtigt werden: In Frankreich sind vor allem die Monate Juli und August Urlaubsmonate und deswegen für den Aufbau von Netzwerken und zum Besuch von Forschungsseminaren nicht geeignet. Dafür ist es in den Bibliotheken und Archiven oft etwas ruhiger und man kommt häufiger mit anderen ausländischen Forschenden ins Gespräch, die die Sommerferien ebenfalls zu Forschungszwecken nutzen.

3 Von der Aufenthaltsdauer hängt auch die Vorbereitung der praktischen Details ab, wie etwa Wohnungssuche (Untermiete auf Tages-, Wochen- oder Monatsbasis oder langfristige Miete), Krankenversicherung, Bankkonto etc. Prinzipiell gilt: je kürzer die Aufenthaltsdauer, desto wichtiger ist eine präzise Planung. 


\section{Vorbereitungen für das alltägliche Leben}

\section{a) Finanzierung}

Da ein längerfristiger Aufenthalt häufig mit einer finanziellen Mehrbelastung verbunden ist, muss als Erstes die Finanzierung sichergestellt werden. Es empfiehlt sich, eine grobe Kostenkalkulation zu erstellen. Vor allem Paris ist sehr teuer - nicht nur der Wohnraum, sondern auch Lebensmittel, essen gehen etc. Für Stipendien gilt es, die Bewerbungsfristen frühzeitig im Auge zu behalten. Oft sind lange Vorlaufzeiten einzukalkulieren, die bis zu einem Jahr betragen können und durch die aufwändigen Auswahlverfahren bedingt sind ${ }^{2}$. Neben den einzelnen Stipendien ist es in Frankreich möglich, zur Deckung der Mietkosten Wohngeldbeihilfe zu beantragen ${ }^{3}$.

5 Nach der Rückkehr vom Forschungsaufenthalt muss eventuell - je nach Finanzierungsart und fördernder Institution - ein Bericht verfasst werden, der über die Verwendung des Stipendiums Rechenschaft ablegt, zur Reflexion der gemachten Erfahrungen einlädt und über Arbeitsfortschritte Auskunft gibt. Teilweise können solche Berichte früherer Stipendiaten bei den entsprechenden Institutionen eingesehen werden und somit auch als Hilfestellung zur Vorbereitung eines Auslandsaufenthalts dienen.

\section{b) Krankenversicherung}

6 Auch bezüglich der Krankenversicherung sollte man sich frühzeitig informieren. Wenn nur ein kurzer Aufenthalt geplant ist, kann man sich mit der europäischen Krankenversichertenkarte begnügen (rechtzeitig bei der Heimatkrankenversicherung beantragen). Es empfiehlt sich außerdem eine Zusatzversicherung abzuschließen, die mögliche Kostendifferenzen zwischen dem deutschen und französischen System übernimmt. Abhängig von der Aufenthaltsdauer gibt es die Möglichkeit, verschiedene Angebote von privaten Zusatzversicherern in Anspruch zu nehmen. Im Internet können diesbezüglich auf den einschlägigen Plattformen wie Stiftung Warentest Vergleiche gefunden werden. Wenn es sich um einen Forschungsaufenthalt im Rahmen eines Masteroder Promotionsstudiengangs handelt, ist zu überlegen, ob man sich an der französischen Universität einschreibt und versichert (bei der LMDE bzw. SMEREP) ${ }^{4}$, denn die Krankenkassenbeiträge sind für Studierende in Frankreich deutlich niedriger als in Deutschland. Die Programmbeauftragten der binationalen Studiengänge der DeutschFranzösischen Hochschule (DFH) können sehr nützliche Informationen hierzu geben. Im Krankheitsfall gibt es darüber hinaus in Paris und Umgebung eine Reihe deutschsprachiger Ärzte, die man kontaktieren kann, falls man sich sprachlich nicht sicher genug fühlen sollte ${ }^{5}$.

\section{c) Wohnungssuche}

7 Vor allem in Paris ist die Wohnungssuche schwierig und langwierig. Deswegen sollte man frühzeitig die Augen offen halten. Während bei kürzeren Forschungsaufenthalten, die nur wenige Tage oder Wochen dauern, immer die Möglichkeit besteht, in einer Jugendherberge ${ }^{6}$, im Hotel oder bei Privatpersonen unterzukommen, übersteigt diese Art der Unterbringung bei längerer Aufenthaltsdauer rasch das finanziell Ertragbare. 
Deswegen müssen für längere Aufenthalte mittel- oder langfristige Lösungen gefunden werden.

8 Generell ist die Wohnungssuche heute in Frankreich zu weiten Teilen internetbasiert. Verschiedene Internetseiten wie pap.fr oder airbnb.com können bei der Wohnungssuche behilflich sein ${ }^{7}$. Es empfiehlt sich immer zu prüfen, ob es nicht neue Webseiten gibt. Die "Klassiker« sind die meist frequentierten Seiten und erhöhen somit die Zahl der möglichen Mitbewerber. Wohngemeinschaften sind in Frankreich seltener als in Deutschland, aber langsam holt man jenseits des Rheins auf ${ }^{8}$.

Die einfachste Lösung ist, eine Wohnung von einem Verwandten oder Bekannten zu übernehmen. Dies ist natürlich schwierig, wenn man gerade erst in der Stadt ankommt und nur über wenig oder keine Kontakte vor Ort verfügt. Im Rahmen der strukturierten deutsch-französischen Förderprogramme für Master und Promotion ${ }^{9}$ besteht aber die Möglichkeit, sich zu erkundigen, ob nicht jemand momentan in Frankreich ist, der sein Zimmer oder seine Wohnung nach Ablauf seines Studien- oder Forschungsaufenthalts abtreten möchte. Eine Wohnungsübernahme hat außerdem den Vorteil, dass die Mietanhebungen häufig geringer ausfallen. Auch über die sozialen Netzwerke kann erfragt werden, ob nicht die Möglichkeit besteht, eine Wohnung zu übernehmen. Auf Facebook existiert beispielsweise eine Gruppe von Deutschen in Paris ${ }^{10}$. Häufig sind die französischen Vermieter relativ misstrauisch, insbesondere gegenüber ausländischen Mietern. Deswegen ist eine Weiterempfehlung durch den Vormieter immer ein guter Ausgangspunkt, vorausgesetzt, dass das bisherige Mietverhältnis problemfrei war.

In Paris selbst kann man beispielsweise beim Goethe-Institut Paris, der Église américaine oder der Église suédoise nach Aushängen schauen ${ }^{11}$. Auch im DHI Paris ${ }^{12}$, dem Deutschen Forum für Kunstgeschichte ${ }^{13}$, der Deutschen Schule Paris ${ }^{14}$ und der Deutschen Botschaft gibt es Ordner bzw. ein schwarzes Brett, wo entsprechende Anzeigen gefunden werden können. Manchmal finden sich Anzeigen von wohlsituierten Familien (v. a. am GoetheInstitut), die Wohnraum gegen Deutschnachhilfe bzw. -unterricht bieten. Wenn die eigene Finanzierung knapp bemessen ist, kann dies eine gute Sparmöglichkeit sein.

11 Historikerinnen und Historiker können Wohnungsanzeigen auf den einschlägigen Fachplattformen im Internet suchen bzw. dort eigene Wohnungsgesuche inserieren (beispielsweise H-Soz-Kult oder H-France) ${ }^{15}$. Außerdem gibt es verschiedene deutschfranzösische Netzwerke, die Anzeigen veröffentlichen (beispielsweise die deutschfranzösische Doktorandengruppe GIRAF-IFFD) ${ }^{16}$. Darüber hinaus ist auch immer an das französische Studentenwerk (CROUS) und die dort angebotenen Unterbringungsmöglichkeiten in Wohnheimen (Cités universitaires) etc. zu denken ${ }^{17}$. Wenn man sich um einen Wohnheimplatz bewirbt, gilt es wiederum, sich frühzeitig um die Bewerbungsunterlagen zu kümmern, da auch hier die Vorlaufzeiten und Wartelisten oft lang sind.

12 Außerhalb der Netzwerke im Bekanntenkreis und des universitären Sektors gibt es natürlich zahlreiche Möglichkeiten, sich auf dem freien Wohnungsmarkt kundig zu machen. Vor allen in Paris selbst laufen viele Angebote über Immobilienmakler, deren Anzeigen großteils auf Plattformen wie seloger.com oder avendrealouer.fr zusammengefasst $\operatorname{sind}^{18}$. Die Konkurrenz bei der Wohnungssuche ist sehr groß und Wohnungsbesichtigungen sind in Paris fast immer Massenveranstaltungen. Es lohnt sich deswegen, einen E-Mail-Alarm einzurichten, um über neue Angebote direkt informiert zu werden, die den persönlichen Suchkriterien entsprechen. Oft sind die Anrufbeantworter 
schon nach einer Stunde voll und man kann nicht einmal mehr Name und Telefonnummer hinterlassen. Zu jeder Wohnungsbesichtigung sollte man das sogenannte dossier mitbringen, bestehend aus verschiedenen Unterlagen wie der Offenlegung der Einkommensverhältnisse und häufig auch einer Bürgschaft (caution solidaire), die Eltern oder Freunde übernehmen können ${ }^{19}$. Immer häufiger werden in Frankreich von den Vermietern Versicherungspolicen gegen Mietausfälle abgeschlossen (assurance loyers impayés), die Ausländer den Zugang zum Wohnungsmarkt erschweren. Die Maklergebühren sind dagegen im Regelfall niedriger als in Deutschland. Je nach Forschungszweck sollte man bei der Wohnungswahl u.a. auf die Verkehrsanbindung achten. Lange Anfahrtswege im morgendlichen und abendlichen Berufsverkehr sind v. a. in Paris meist sehr zeit- und nervenraubend.

Bei einem längeren Aufenthalt in Frankreich sollte auch über die Einrichtung eines französischen Bankkontos nachgedacht werden. Wer ein Stipendium in Frankreich einwerben will, braucht ein französisches Konto, da die Stipendien nicht auf ausländische Konten überwiesen werden. Auch wenn heute über verschiedene Kooperationen zwischen den Banken in Europa nicht mehr grundsätzlich Gebühren für ECKartenzahlungen und Bargeldabhebungen anfallen, erleichtert ein französisches Bankkonto doch vieles: Beispielsweise ist in Frankreich bis heute der Scheck ein gebräuchliches Zahlungsmittel, und die Kaution für Wohnungen wird häufig in dieser Form hinterlegt. Auch für die Strom- und Gasrechnung empfiehlt es sich, ein französisches Konto mit Bankeinzugsermächtigung einzurichten. Darüber hinaus dient die Gas-, Strom- oder Telefonrechnung als Nachweis über den jeweiligen Wohnsitz und ersetzt, da es in Frankreich keine polizeiliche Meldepflicht gibt, die deutsche Einwohnermeldebescheinigung. Insbesondere in Ausleihbibliotheken wird eines dieser drei Dokumente als Nachweis verlangt, um die Berechtigung zur Buchausleihe $\mathrm{zu}$ erhalten. Ein französisches Bankkonto ist auch wichtig, wenn man die Unterstützung der Caisse d'allocation familiales (CAF) in Anspruch nehmen will. Diese Einrichtung übernimmt für Studierende - je nach Einkommenslage - einen Teil der Mietkosten, was vor allem in Paris sehr wichtig werden $\mathrm{kann}^{20}$. Apropos Kosten: Der Telefon- und Mobilfunkmarkt ist in Frankreich stärker durch offene Konkurrenz geprägt. Die verschiedenen Angebote enthalten deswegen deutlich weniger versteckte Kosten, und meist bekommt man im Vergleich zu Deutschland mehr Leistung für den gleichen Preis ${ }^{21}$.

\section{d) Sprachschulen und Jobmöglichkeiten}

14 Wer vorbereitend für den Forschungsaufenthalt noch seine Französisch-Kenntnisse auffrischen will, kann einen der vielen Sprachkurse besuchen, die sowohl von privaten als auch von staatlichen Sprachschulen angeboten werden ${ }^{22}$. Auf das Wissenschaftsfranzösisch kann man sich beispielsweise gezielt durch einen Kurs am DHI Paris vorbereiten ${ }^{23}$. Die internationalen Abteilungen der Universitäten sind ebenfalls in der Lage, eine Liste der entsprechenden Möglichkeiten zu geben, zumal die Universitäten oftmals selbst Kurse anbieten, die die notwendigen Fachsprachenkenntnisse für den Wissenschaftsbereich vermitteln.

Nebenjobmöglichkeiten bestehen unzählige. Wer noch nicht gut Französisch spricht, kann sich beispielsweise in einen Call Center bewerben (Betreuung deutscher Kunden) oder ganz klassisch als Kellner arbeiten. Darüber hinaus gibt es die Möglichkeit, bezahlte Archivrecherchen zu übernehmen. Die entsprechenden Anzeigen werden oft auf H- 
France bzw. in der kostenlosen englischsprachigen Zeitschrift $» F u s a c «^{24}$ publiziert. Anders als in Deutschland, wo zahlreiche studentische und wissenschaftliche Hilfskraftstellen angeboten werden, kennt das französische Hochschul- und Wissenschaftssystem kaum solche Nebenverdienstmöglichkeiten, die Geldverdienen und Vernetzung in der Wissenschaft zugleich ermöglichen.

\section{Forschungsorganisatorisches}

\section{a) Kontaktaufnahme mit Forschenden}

16 Zur Vorbereitung eines Forschungsaufenthalts empfiehlt es sich für Historikerinnen und Historiker ganz besonders, das Deutsche Historische Institut (DHI) in Paris zu kontaktieren, um schnell weiterführende Informationen zu erhalten ${ }^{25}$. Die dort tätigen Wissenschaftlerinnen und Wissenschaftler sind mit der französischen Archiv- und Forschungslandschaft bestens vertraut und können den Einstieg durch persönlichen Rat bzw. die Weiterleitung der entsprechenden Anfrage an französische Kolleginnen und Kollegen deutlich erleichtern. Der direkte Kontakt bereichert immer die Forschungsarbeit. Die französischen Historikerinnen und Historiker kennen oft die Archive und ihre Bestände besonders gut, bedingt durch den starken Quellenbezug im französischen Masterstudium. Darüber hinaus bietet das DHI Paris regelmäßig Kurse zur Einführung in das französische Wissenschaftssystem (gemeinsam mit dem CIERA) ${ }^{26}$ sowie Französisch als Wissenschaftssprache (gemeinsam mit dem Deutschen Forum für Kunstgeschichte) ${ }^{27}$ an $^{28}$. Weitere Hilfestellung für die Kontaktaufnahme bieten in Paris darüber hinaus das vom DAAD geförderte CIERA (Centre interdisciplinaire d'études et de recherches sur l'Allemagne) und das Heinrich-Heine-Haus ${ }^{29}$ in der Cité universitaire sowie manche Goethe-Institute.

Die Kontaktaufnahme mit den Universitätsprofessorinnen und -professoren ist in Frankreich - gerade für Masterstudierende oder Promovierende - teilweise stark formalisiert und unterliegt häufig impliziten Regeln. Es gibt durchaus Professoren, die eher selten auf E-Mails von Unbekannten reagieren, aber rasch auf handgeschriebene Briefe, es findet sich aber auch das Gegenteil. Auch hier können persönliche Kontakte oder die Vermittlung durch Dritte hilfreich sein. Die Wahl des richtigen Kommunikationskanals kann den Kontaktaufbau deutlich erleichtern. Nicht immer handelt es sich um Arroganz oder Desinteresse, wenn man keine Antwort bekommt. Darüber hinaus ist es möglich, sobald man in Frankreich ist, Lehrveranstaltungen und Seminare zu besuchen und sich dort persönlich vorzustellen.

18 Die französischen Historikerinnen und Historiker organisieren sich in vier großen Verbänden. Ein Dachverband, der dem deutschen Historikerverband vergleichbar wäre, existiert nicht. Auf den jeweiligen, teilweise noch im Aufbau befindlichen Webseiten finden sich häufig die Kontaktdaten der Forscher ${ }^{30}$. Auf den Webseiten werden darüber hinaus Tagungen und Neuveröffentlichungen angekündigt. Parallel dazu sind die Anzeigen auf calenda.org für Seminare, Workshops, Kolloquien etc. im Auge zu behalten, die es ermöglichen, sich über die neueste Forschung zu informieren ${ }^{31}$. Eine Übersicht der laufenden Doktorarbeiten und deren Betreuer findet sich auf der Internetseite www.theses.fr. ${ }^{32}$

19 Wer an eine Masterarbeit in einem deutsch-französischen Studiengang bzw. eine thèse en cotutelle für die Promotion denkt, sollte darauf achten, dass nicht nur thematisch und 
methodisch der Bezug zum Betreuer stimmt, sondern auch der persönliche Kontakt ${ }^{33}$. Die Wahl des Betreuers kann in Deutschland wie in Frankreich Zukunftschancen in bestimmten Bereichen eröffnen bzw. verbauen. Oft handelt es sich dabei um implizites Wissen, das man nur durch einen längeren Aufenthalt erlangen kann. Die entsprechenden Konstellationen werden häufig erst nach langer Aufenthaltsdauer verständlich. Die Einschätzungen sind immer subjektiv und nicht selten durch persönlichen Erfolg bzw. Misserfolg gefärbt.

Die Tragweite der Wahl der Universität und des Betreuers für die weitere berufliche Laufbahn darf nicht unterschätzt werden. Es gilt hier schon früh ein Gespür für die entsprechenden Mechanismen zu entwickeln. Gerade wer sich langfristig eine Karriere in Frankreich vorstellen kann, sollte versuchen, in diesem Bereich besonders aufmerksam zu sein. Die französische Hochschullandschaft ist im Bereich der Geschichtswissenschaft noch stärker als die deutsche von einer offenen Politisierung gekennzeichnet. Entsprechend sind die Karrierechancen teilweise durch außerfachliche Faktoren bestimmt. Während es sich in Deutschland häufig um personenzentrierte Netzwerke handelt, spielt in Frankreich die Institution eine stärkere Rolle ${ }^{34}$. So gibt es universitäre Netzwerke, die ihre Kandidaten gegenseitig rekrutieren und Qualitätskriterien dabei mitunter zurückstellen ${ }^{35}$.

\section{b) Anfragen an die Bibliotheken und Archive}

21 Durch die zunehmende Digitalisierung im Bereich des Archiv- und Bibliothekswesens kann man Forschungsaufenthalte heute wesentlich einfacher und präziser von Deutschland aus in Eigenregie vorbereiten. Zwar ist ein Schreiben (oder eine E-Mail) an das jeweilige Archiv oder die Bibliothek immer noch überaus sinnvoll, aber bereits am heimischen Computer lassen sich heute wichtige Informationen zu den Beständen und Standorten von Archivalien, Büchern und sonstigen Quellen sammeln. Wie immer gilt auch hier: Je präziser die Anfrage ist, desto mehr Information erhält man von den Zuständigen aus den Archiven bzw. Bibliotheken. Häufig reagieren die französischen Archive und Bibliotheken sehr freundlich und hilfsbereit auf die Anfragen.

Die Archive und Bibliotheken verfügen heute oftmals über digitalisierte Findmittel, entweder in Form von Datenbanken oder zumindest in Form von $\mathrm{PDF}^{36}$. Die Tiefenerschließung in Form von Digitalisierung ist nicht überall gleich weit fortgeschritten. Es ist aber fast immer möglich, die Fundorte in den Archiven zu ermitteln. Darüber hinaus sind die einschlägigen Findmittel der großen Institutionen wie der Manuskriptsammlung der Bibliothèque nationale de France (BnF) oder des Außenministeriums auch in Buchform erschienen und können in den deutschen Bibliotheken eingesehen werden. Bei Inventaren, die der grauen Literatur angehören, ist dies leider nicht möglich. Wenn kein Scan vorliegt, muss man die physischen Bände vor Ort einsehen und auswerten, was bei der Zeitplanung einkalkuliert werden muss.

Der in Frankreich vielfach praktizierte Zentralismus vereinfacht oft die Suche: Beispielsweise lassen sich die Bestände $\mathrm{zu}$ Archivalien in französischen Universitätsbibliotheken und anderen Forschungseinrichtungen über die Plattform CALAMES recherchieren ${ }^{37}$. Gleiches gilt auch für die Suche nach Büchern und seltenen Drucken, deren Standorte über den »Catalogue collectif de France« (cCfr) bzw. den Sudoc ermittelt werden können ${ }^{38}$. 

behalten. Die BnF-Site Mitterand schließt beispielsweise Anfang September, das Archiv des Außenministeriums Ende April. Auf den jeweiligen Webseiten sind diese Informationen leicht zugänglich. Auch sollte man sich im Voraus über die Möglichkeit von Reproduktionen in Form von Fotografien, Kopien, Mikroverfilmungen etc. informieren. Je nach Institution können dabei beträchtliche Kosten entstehen. So versteht sich beispielsweise die BnF in erster Linie als Aufbewahrungsort von französischem Kulturgut. Um diesem Auftrag gerecht zu werden, sind die Preise für Reproduktionen sehr hoch. Dies verhindert unnötige Kopien und schützt die Buchbindung. In manchen Fällen kann man bei den Lehrstühlen zu Hause oder bei Stipendiengebern etc. anfragen, ob für Reproduktionen Geld bereitgestellt werden kann. Es muss hier immer zwischen Aufenthalts- und Reproduktionskosten abgewogen werden (kurzer Aufenthalt mit vielen Kopien, die erst zu Hause ausgewertet werden, oder langer Aufenthalt, der die Quellen im Archiv erschließt).

25 Auch ist es sinnvoll, frühzeitig die Zugangsbedingungen $\mathrm{zu}$ den einzelnen Archivbeständen und Bibliotheken zu erfragen. Je nach Forschungsgegenstand können Empfehlungsschreiben von bekannten Professoren den Zugang erleichtern bzw. erst ermöglichen. Auch für den Forschungsbereich der BnF ((nach ihrer Lage »Rez-de-jardin« genannt) sollten Studierende und Promovierende ein auf französisch-verfasstes Empfehlungsschreiben zur Einschreibung mitbringen. Die Bibliothekare hüten die dortigen Schätze und wollen nur bei wirklichem Forschungsinteresse den Zutritt gewähren.

\section{c) Vorlesungen und Forschungsseminare in Frankreich}

26 Je nach Aufenthaltsort findet man in Frankreich ein sehr reichhaltiges Angebot an Lehrveranstaltungen und Forschungsseminaren vor $^{39}$. Geschichte hat in Frankreich gesellschaftlich einen wesentlich höheren Stellenwert als in Deutschland ${ }^{40}$. Deswegen gibt es oftmals deutlich mehr Veranstaltungen an den Universitäten. Das Nebeneinander von grandes écoles, Universitäten und den jeweiligen Forschungsinstituten des CNRS (Centre National de la Recherche Scientifique) vergrößert das Angebot beträchtlich ${ }^{41}$. Insbesondere in Paris kann man täglich aus einer Vielzahl an Vorträgen, Seminaren, Vorlesungen sowie Tagungen auswählen. Neben den Universitäten sind die Veranstaltungen der Archive, des Collège de France, der École des Hautes Études en Sciences Sociales (EHESS), der École Pratique des Hautes Études (EPHE), der École Normale Supérieure (ENS), aber auch verschiedener Buchhandlungen zu nennen, die häufig namhafte Historikerinnen und Historiker einladen. Doch auch in den Regionen wird man sich nicht langweilen: Veranstaltungen organisieren etwa die Maisons des Sciences de l'Homme, die jeweiligen Universitäten und die einzelnen geschichtswissenschaftlichen Forschungsinstitute, die als Unités mixtes de recherche (UMR) oft gemeinsam mit dem CNRS betrieben werden sowie historische Museen ${ }^{42}$.

27 Auch wenn die Seminarform, die in Frankreich ab dem Master überwiegt, nicht mit der deutschen Seminarkultur zu vergleichen ist, ermöglichen die jeweiligen Veranstaltungen doch gute Einblicke in die akademische Kultur Frankreichs. Die Diskussionskultur, die Art des Nachfragens, der gezielte Einsatz von Rhetorik etc. können in diesem Rahmen durch aufmerksames Beobachten gut studiert und gelernt werden. Dabei handelt es sich ebenso zumeist um implizites (kulturelles und soziales) Wissen, das nur durch die Teilnahme an 
den entsprechenden Veranstaltungen erworben werden kann. Allein schon zum Erlernen des geschichtswissenschaftlichen Fachvokabulars und der gängigen Floskeln zur Einleitung von Fragen oder Kritik lohnt sich der Besuch in verschiedenen Veranstaltungen.

\section{Schluss}

Die deutsche und die französische Wissenschaftskultur haben beide ihre Stärken und Schwächen. Nur durch den Vergleich kann man sich der Eigenheiten der jeweiligen Wissenschaftssysteme bewusst werden. Wichtig ist es, nicht das eine im Vergleich zum anderen als defizitär zu beschreiben. Häufig sind deutsche Historiker vom meist etwas verschulten System in Frankreich enttäuscht, genauso wie französische Historiker oft von der eher abstrakten Diskussionskultur in Deutschland abgeschreckt sind ${ }^{43}$. Die deutsche Geschichtswissenschaft ist aber nicht besser, sondern einfach anders - und genau dies gilt es zu begreifen. Wer im deutsch-französischen Bereich Fuß fassen will, sollte deswegen versuchen, die Regeln für beide Wissenschaftssysteme langfristig zu erlernen. Dies geht notwendigerweise mit einer zeitweisen Vermischung der beiden akademischen Traditionen einher. Langfristig sollten sowohl die deutschen als auch die französischen Regeln der Historikerzunft beherrscht werden, um diese gezielt als solche im jeweiligen Diskursraum einsetzen zu können ${ }^{44}$.

\section{NOTES}

1. Vgl. dazu die Beiträge von Pierre Monnet "Institutionen und Strukturen der Geschichtswissenschaft in Frankreich" (http://ifha.revues.org/7846) und Ulrike Krampl "Geschichte studieren in Frankreich« in diesem Wegweiser (http://ifha.revues.org/7851). Alle Links wurden zuletzt am 15.07.2014 überprüft.

2. Vgl. dazu den Beitrag von Delia Küsgen und Christiane Schmeken in diesem Wegweiser »Die Finanzierung eines Studien-, Lehr- und Forschungsaufenthaltes in Frankreich « (http:// ifha.revues.org/7919).

3. Vgl. den Abschnitt »Wohnungssuche« in diesem Beitrag.

4. Vgl. dazu auch den Beitrag von Ulrike Krampl »Geschichte studieren in Frankreich« in diesem Wegweiser, Abschnitt 25 (http://ifha.revues.org/7851).

5. Deutsche Botschaft Paris, http://www.allemagne.diplo.de/contentblob/3524904/ Daten/3912111/02medecinsdatei.pdf.

6. Auberges de jeunesse, http://www.aubergesdejeunesse.com/France/Paris.

7. Vgl. beispielsweise für Privatanzeigen: De Particulier à Particulier, http://www.pap.fr/, für kurzfristige Mieten beispielsweise Airbnb, https://www.airbnb.de/.

8. Vgl. beispielsweise die beiden Seiten Colocation.fr, http://www.colocation.fr und Appartager, http://www.appartager.com. Parallel dazu entwickeln sich neue Formen, wie sie hier am Beispiel Paris vorgestellt werden: Lebonbon, http://www.lebonbon.fr/Les-Tops/Comment-trouver-unecolocation-a-Paris. 
9. Vgl. dazu allgemein die Beiträge von Ulrike Krampl »Geschichte studieren in Frankreich« in diesem Wegweiser (http://ifha.revues.org/7851) sowie von Falk Bretschneider und Christophe Duhamelle »Promovieren in Geschichte und die cotutelle in Frankreich" (http:// ifha.revues.org/7868). Zur Doktoranden-Förderung auch die Liste der deutsch-französischen $\mathrm{H}$ ochschule http://www.dfh-ufa.org/uploads/media/Tableaux_DFDK_CDFA_2014.pdf.

10. Facebookgruppe »Deutsche in Paris«, https://www.facebook.com/groups/deutscheinparis/.

11. Vgl. Etudiantdeparis, http://www.etudiantdeparis.fr/node/95.

12. Deutsches Historisches Institut Paris, http://www.dhi-paris.fr.

13. Deutsches Forum für Kunstgeschichte, http://www.dtforum.org/.

14. Deutsche Schule Paris, http://www.idsp.fr/.

15. http://hsozkult.geschichte.hu-berlin.de/chancen/type=wohnungen und http://h-france.net/ housingdigest.html. Die Mieten der auf H-France angebotenen Wohnungen sind häufig höher als normal.

16. Vgl. Facebookgruppe »GirafIffd«, https://www.facebook.com/GirafIffd.

17. Vgl. beispielsweise für Paris: CROUS Paris, http://www.crous-paris.fr/article.asp?idcat=AAD u nd Cité internationale universitaire de Paris, http://www.ciup.fr/maisons/houses-admission/.

18. Se loger, http://www.seloger.com/ und Avendrealouer, http://www.avendrealouer.fr.

19. Vgl. zum dossier beispielsweise: De Particulier à Particulier, http://www.pap.fr/conseils/ location/locataire-constituez-un-bon-dossier/a1779. Vorlagen für die caution solidaire finden sich auf verschiedenen Seiten im Internet.

20. Allocations familiales, http://www.caf.fr/ und der Beitrag von Ulrike Krampl »Geschichte studieren in Frankreich« in diesem Wegweiser, Abschnitt 26 (http://ifha.revues.org/7851).

21. Vgl. beispielsweise die kurze deutschsprachige Übersicht: Campus France, http:// www.allemagne.campusfrance.org/node/93605.

22. Vgl. dazu beispielsweise: Fachverband Deutscher Sprachreise-Veranstalter e. V., h ttp://www.fdsv.de/sprachreisenfinder-liste.html?no_cache=1 und die Übersicht auf der Seite des Deutschen Akademischen Austausch Dienstes, https://www.daad.de/ausland/ sprachen-lernen/sprachkurse/de/476-sprachkurse-an-hochschulen-in-ausgewaehltenlaendern/.

23. Zum Vergleich der Wissenschaftssprache vgl. den Beitrag von Anne Baillot und Franziska Heimburger "Geschichte schreiben: Französisch als Wissenschaftssprache« in diesem Wegweiser (http://ifha.revues.org/7931). Außerdem: Dirk Siepmann, Academic Writing and Culture: An Overview of Differences between English, French and German, in: Meta. Journal des traducteurs 51,1 (2006), S. 131-150, insbesondere der tabellarische Überblick auf S. 142f.

24. Fusac, http://www.fusac.info/last/index.html.

25. DHI Paris, http://www.dhi.fr/.

26. CIERA, http://www.ciera.fr/ciera/?lang=de.

27. Deutsches Forum für Kunstgeschichte (DFK), http://www.dtforum.org/.

28. Vgl. den Herbstkurs 2013: „Einführung in die Wissenschaftssprache Französisch und in die Forschungspraxis« (16.-24. September 2013). Vgl. auch die Beiträge von Pierre Monnet (http:// ifha.revues.org/7846), Ulrike Krampl (http://ifha.revues.org/7851), Falk Bretschneider und Christophe Duhamelle (http://ifha.revues.org/7868), Jean-Louis Georget (http:// ifha.revues.org/7940), Anne Baillot und Franziska Heimburger in diesem Wegweiser (http:// ifha.revues.org/7931).

29. Heinrich-Heine-Haus, http://www.maison-heinrich-heine.fr/fr/.

30. Vgl. die Webseiten der Historiker der Antike: Société des Professeurs d'Histoire Ancienne à l'Université, http://sophau.univ-fcomte.fr/, der Mediävistik: Société des Historiens Médiévistes de l'Enseignement Supérieur Public, http://www.shmesp.fr/, der Frühen Neuzeit: Association des Historiens Modernistes des Universités Françaises, http://ahmuf.hypotheses.org/ und der 
neueren und neusten Geschichte: Carnet de l'Association des historiens contemporanéistes de l'enseignement supérieur et de la recherche, http://ahcesr.hypotheses.org/. Vgl. zu den Organisationsstrukturen auch allgemein der Beitrag von Pierre Monnet »Institutionen und Strukturen der Geschichtswissenschaft in Frankreich" in diesem Wegweiser (http:// ifha.revues.org/7846).

31. Calenda, http://calenda.org.

32. These.fr, http://www.theses.fr/.

33. Zur thèse en cotutelle vgl. den Beitrag von Falk Bretschneider und Christophe Duhamelle "Promovieren in Geschichte und die cotutelle in Frankreich" in diesem Wegweiser (http:// ifha.revues.org/7868).

34. Zu den Berufsaussichten für Historiker in Frankreich vgl. den Beitrag von Jean-Louis Georget »Karrieremöglichkeiten in der Wissenschaft und der Arbeitsmarkt für Historikerinnen und Historiker in Frankreich« in diesem Wegweiser (http://ifha.revues.org/7940).

35. Vgl. dazu die verschiedenen Beiträge auf Academia, http://academia.hypotheses.org/. Das Verfahren hat in den letzten Jahren durch ein Wiki an Transparenz gewonnen, vgl. Association Française d'Histoire économique, http://afhe.hypotheses.org/suivi-des-recrutements.

36. Vgl. dazu auch die Beiträge zu den Bibliotheken von Dominique Bouchery und Mareike König "Bibliotheken in Frankreich: Einrichtungen, Bestände und Suchstrategien« (http:// ifha.revues.org/7889) sowie den Beitrag zu den Archiven von Matthias Nuding und Florence de Peyronnet-Dryden »Archive in Frankreich« in diesem Wegweiser (http://ifha.revues.org/7877).

37. Catalogue en Ligne des Archives et des Manuscrits de l'Enseignement Supérieur, http:// www.calames.abes.fr/pub/.

38. Catalogue collectif de France, http://ccfr.bnf.fr/ und SUDOC, http://www.sudoc.abes.fr/.

39. Vgl. das immer noch aufschlussreiche Kapitel »Mit fremden Augen gesehen. Erfahrungen an französischen Universitäten« aus der Sicht eines deutschen Dozenten an der französischen Universität in: Karl Heinz Götze, Französische Affären. Ansichten von Frankreich, Frankfurt a.

M. 1993, S. 59-102.

40. Vgl. dazu auch den Beitrag von Nicolas Offenstadt in diesem Wegweiser »Die französische Geschichtsschreibung am Anfang des 21. Jahrhunderts - einige Einblicke« (http:// ifha.revues.org/7835).

41. Vgl. dazu den Überblick bei Pierre Monnet »Institutionen und Strukturen der Geschichtswissenschaft in Frankreich« in diesem Wegweiser (http://ifha.revues.org/7846).

42. Vgl. beispielsweise für Lyon: http://triangle.ens-lyon.fr/.

43. Vgl. zur Rezeption beider Wissenschaftskulturen: Gerd Krumeich, Thomas Beckers, Zeitgeschichte aktuell? Transfer von Deutschland nach Frankreich, in: Fritz Nies (Hg.), Spiel ohne Grenzen? Zum deutsch-französischen Transfer in den Geistes- und Sozialwissenschaften, Tübingen 2002, S. 97-124 und Gabriele Lingelbach, Geringe Wahrnehmung als Tradition. Transfer von Deutschland nach Frankreich, in: Ibid., S. 125-150.

44. Vgl. die immer noch anregenden und zugleich amüsanten Überlegungen zu den verschiedenen Wissenschaftsstilen von Johan Galtung, Struktur, Kultur und intellektueller Stil. Ein vergleichender Essay über sachsonische, teutonische, gallische und nipponische Wissenschaft, in: Alois Wierlacher (Hg.), Das Fremde und das Eigene. Prolegomena zu einer interkulturellen Germanistik, München 1985, S. 151-193. 


\section{RÉSUMÉS}

Zur Vorbereitung eines Forschungsaufenthalts in Frankreich müssen sowohl lebenspraktische als auch forschungsorganisatorische Aspekte berücksichtigt werden. Der erste Teil des Artikels behandelt Fragen wie Krankenversicherung, Wohnungssuche, Bankkontoeinrichtung etc. Der zweite Teil geht spezifisch auf die Forschungsorganisation ein und versteht sich als Hilfestellung für die Kontaktaufnahme mit französischen Kolleginnen und Kollegen, Archiven und Bibliotheken. Darüber hinaus werden erste Hinweise zur Forschungslandschaft in Frankreich gegeben.

\section{INDEX}

Schlüsselwörter : Frankreich, Studium, Universität, Alltagsleben, Aufenthalt, Interkulturalität

\section{AUTEUR}

\section{NIELS F. MAY}

Dr. Niels F. May ist Post-Doktorand am Institut français d'histoire en Allemagne (Frankfurt a. M.). Mail: niels.may[at]institutfrancais.de 